\title{
Ultrathin porphyrin and tetra-indole covalent organic frameworks for organic electronics applications
}

Cite as: J. Chem. Phys. 153, 044702 (2020); https://doi.org/10.1063/5.0010164

Submitted: 07 April 2020 . Accepted: 01 July 2020 . Published Online: 22 July 2020

Orestis George Ziogos (D), Itsaso Blanco (D), and Jochen Blumberger (D)

\section{COLLECTIONS}

Paper published as part of the special topic on 2D Materials

Note: This paper is part of the JCP Special Topic on 2D Materials.
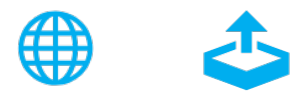

\section{ARTICLES YOU MAY BE INTERESTED IN}

Polarizable charges in a generalized Born reaction potential

The Journal of Chemical Physics 153, 024111 (2020); https://doi.org/10.1063/5.0012022

Optical properties of charged excitons in two-dimensional semiconductors

The Journal of Chemical Physics 153, 034703 (2020); https://doi.org/10.1063/5.0012475

Unveiling coupled electronic and vibrational motions of chromophores in condensed phases

The Journal of Chemical Physics 151, 200901 (2019); https://doi.org/10.1063/1.5128388

\section{Lock-in Amplifiers up to $600 \mathrm{MHz}$}
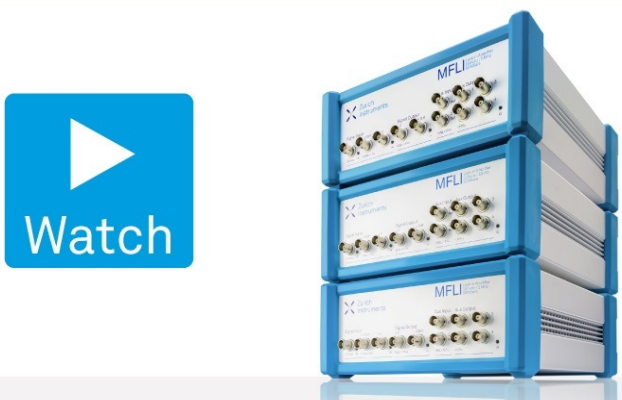


\title{
Ultrathin porphyrin and tetra-indole covalent organic frameworks for organic electronics applications
}

\author{
Cite as: J. Chem. Phys. 153, 044702 (2020); doi: 10.1063/5.0010164 \\ Submitted: 7 April 2020 - Accepted: 1 July 2020 • \\ Published Online: 22 July 2020
}

Orestis George Ziogos, a) (D) Itsaso Blanco, (D) and Jochen Blumberger (D)

AFFILIATIONS

Department of Physics and Astronomy, University College London, Gower Street, London WC1E 6BT, United Kingdom

Note: This paper is part of the JCP Special Topic on 2D Materials.

author to whom correspondence should be addressed: o.ziogos@ucl.ac.uk

\begin{abstract}
The electronic and charge transport properties of porphyrin and tetra-indole porphyrinoid single layer covalent organic frameworks (COFs) are investigated by means of density functional theory calculations. Ultrathin diacetylene-linked COFs based on oxidized tetra-indole cores are narrow gap 2D semiconductors, featuring a pronounced anisotropic electronic band structure due to the combination of dispersive and flat band characteristics, while registering high room temperature charge carrier mobilities. The capability of bandgap and charge carrier localization tuning via the careful selection of fourfold porphyrin and porphyrinoid cores and twofold articulated linkers is demonstrated, with the majority of systems exhibiting electronic gap values between $1.75 \mathrm{eV}$ and $2.3 \mathrm{eV}$. Tetra-indoles are also capable of forming stable monolayers via non-articulated core fusing, resulting in $2 \mathrm{D}$ morphologies with extended $\pi$-conjugation and semi-metallic behavior.
\end{abstract}

Published under license by AIP Publishing. https://doi.org/10.1063/5.0010164

\section{INTRODUCTION}

Covalent organic frameworks (COFs) constitute a diverse and constantly expanding class of porous polymeric materials with promising applications in a plethora of fields, such as gas and liquid storage and separation processes, catalysis, electrochemical energy storage, charge transport (CT), and optoelectronics. ${ }^{1-4}$ An intriguing characteristic of COFs is the capability to tune material properties via the selection of suitable precursor molecules. As a result, a standing challenge is to identify the right combinations of molecular fragments as to optimize specific physicochemical properties.

Amongst their numerous potential applications, COFs have been gradually gaining ground as active media in organic electronics (OEs). ${ }^{5-9}$ Therefore, a pertinent question is which types of precursor molecules could form systems with appropriate properties for such applications. A currently developing class of frameworks with significant implementations in various fields is porphyrin-based COFs. ${ }^{10-20}$ Members of this family of materials have already been employed as candidate COF materials for CT and optoelectronic applications. $^{21}$

This work is focused on the computational screening of ultrathin porphyrin- and porphyrinoid-based COFs for OE applications by means of Density Functional Theory (DFT) calculations. The electronic and CT properties of monolayer COFs made up of either threefold or fourfold core molecules, covalently joined via articulated linkers, have been extensively studied in the computational literature, providing this way a valuable insight regarding complex phenomena and mechanisms. DFT calculations have been successfully employed toward the determination of electronic and optical properties of monolayer COFs in general $^{26-33}$ and in particular for porphyrin- and phthalocyanine-based COFs. ${ }^{34-38}$ Moreover, simulation work in the literature has focused on the examination of in-plane CT mechanisms, either by means of the Boltzmann Transport Equation (BTE) theory ${ }^{39-41}$ or by utilizing mixed quantum-classical dynamics methods. ${ }^{41}$ Furthermore, the special case of planar molecules fused via non-articulated linkers, practically resulting in monolayer holey structures with 
extended $\pi$-conjugation, has been examined in the literature via DFT simulations. ${ }^{4-45}$

The selection of the systems under study is mainly inspired by the phenomenal synthetic versatility of porphyrin-based nanostructures already realized in the literature. ${ }^{46-50}$ In this work, we focus on the determination of electronic and CT properties of ultrathin COF structures based on fourfold metal-free porphyrin $(\mathrm{P})$ and tetra-indole porphyrinoid cores, ${ }^{51,52}$ linked either via variable articulated twofold spacers or through proper aromatic ring fusion as to avoid unfavorable steric hindrances. Tetra-indole porphyrinoids are initially differentiated via alternative peripheral functionalization schemes, resulting in cores with grafting sites on the 5 indole position (abbreviated as TIP in the manuscript) or on the 4 indole position, labeled TIP(4). In addition, a second differentiation arises through the oxidation of the tetra-indole molecule, leading to cores with only two diametrically opposite $\mathrm{N}-\mathrm{H}$ groups, referred to as TIP- $2 \mathrm{H}$ and TIP(4)-2H when utilizing the 5 and 4 indole grafting positions, respectively. The twofold linkers used are diacetylene (Ac2), phenyl ( $\mathrm{Ph})$, pyrene (Py), diphenyl-diacetylene (Ph2Ac2), and the shortest variant $(n=0)$ of the 1,4-terephthalylidene-bis- $N$ $\left(4^{\prime}-n\right.$-alkylaniline) homologous series: TBAA. All molecular fragments are depicted in Fig. 1. In the case of fused TIP-2H cores, a conjugation extension can be achieved via carbon or nitrogen atom bridging on the $(4,5)$ indole positions, defining this way the TIP-COF and TIP-N-COF systems, respectively. Finally, a core fusion route without out-of-plane distortions can be achieved via nitrogen bridging on the $(5,6)$ indole positions, resulting in the TIP-N-COF $(5,6)$ system.

The electronic band structure and CT capabilities of the P-Ac2 prototype COF system have been reported by Thomas et al., 38,41 suggesting semiconducting behavior with a narrow bandgap and high charge carrier mobilities. We take into consideration the most straightforward modification of the P-Ac2 system, based on the replacement of the porphyrin core with tetra-indole variants, as to examine the interplay between different core electronic structures and periodic monolayer properties via DFT simulations. The introduction of different types of tetra-indole cores is found to have a strong effect on the band structure, the energy gap value and type, and parameters governing the charge mobility predicted by the BTE theory. The dependency of the band structure on the twofold linker type is also examined, elucidating this way the tunability of electronic properties upon different core and linker combinations. On a different level of core interconnection, the effect of core fusion via conjugation extensions on electronic and CT properties has been demonstrated by Gutzler ${ }^{43}$ for metal-free phthalocyanine and Pham et al. ${ }^{44}$ for nickel phthalocyanine derivatives, revealing direct-gap semiconducting behavior with sizable charge mobilities. We demonstrate that all fused TIP-2H systems under study exhibit semi-metallic behavior, in contrast to their phthalocyanine analogs.

Our basic findings indicate that ultrathin COFs based on tetraindole derivatives demonstrate tunable semiconducting behavior when articulated twofold linkers are employed, with a wide range of bandgap values and promising room temperature in-plane charge carrier mobilities with interesting anisotropic effects. In the case of direct core fusion via a rigid conjugation extension, fused oxidized tetra-indole monolayers display a semi-metallic character, hence constituting a series of materials with possible applications in the field of 2D synthetic metals. (a)
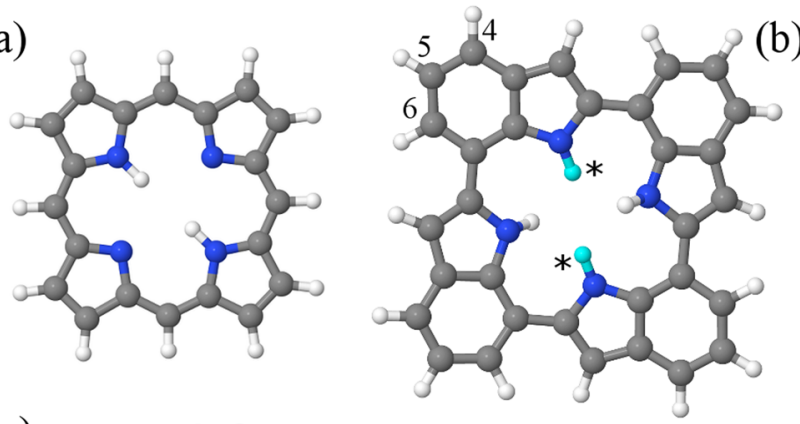

(c)
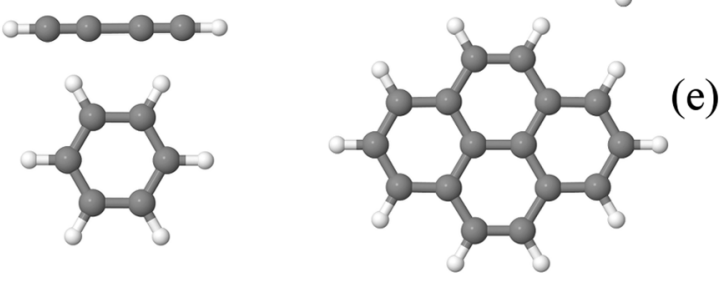

(f)

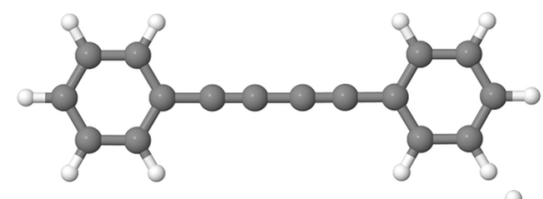

(g)

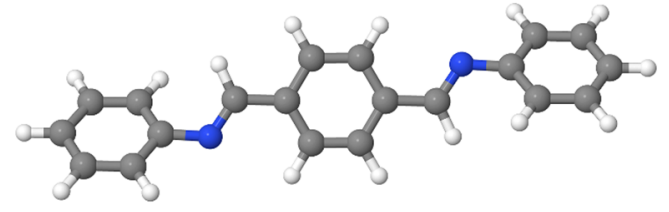

FIG. 1. The building blocks for all monolayer COFs under study: (a) porphyrin and (b) tetra-indole porphyrinoid fourfold cores and (c) diacetylene, (d) phenyl, (e) pyrene, (f) diphenyl-diacetylene, and (g) TBAA twofold linkers. Indole peripheral positions are indicated using the digits 4,5 , and 6 . The hydrogen atoms removed upon oxidation to define the TIP-2H core are highlighted using asterisks.

The layout of the paper is organized follows: technical details on the calculation of electronic and CT properties are outlined in Sec. II, followed by the results in Sec. III. The conclusions of this study are presented in Sec. IV.

\section{COMPUTATIONAL DETAILS}

The structural and electronic characterization of monolayer COFs is carried out by means of plane wave DFT simulations. Core states are treated with ultrasoft pseudopotentials, ${ }^{53}$ with a wavefunction cutoff of $40 \mathrm{Ry}$, except for systems containing nickel, for which the cutoff is set to $80 \mathrm{Ry}$. All periodic structures are equilibrated using the Perdue-Burke-Ernzerhof (PBE) exchange-

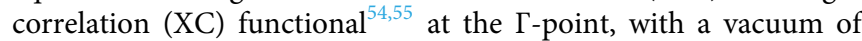
$15 \AA$ along the $z$ direction. Convergence analyses were carried out on both wavefunction cutoff values and $k$-point grids. The electronic density of all equilibrated structures is evaluated using a $5 \times 5 \times 1$ Monkhorst-Pack uniform grid. Electronic density of states (DOS) calculations are carried out with a finer $15 \times 15 \times 1$ grid, 
using the Blöchl tetrahedron method. ${ }^{56}$ All PBE calculations are performed using the Quantum Espresso code. ${ }^{57}$ The electronic properties of selected systems are further refined via the utilization of the Heyd-Scuseria-Ernzerhof (HSE06) range-separated hybrid XC functional $^{58}$ using the CASTEP code. ${ }^{59}$ Single molecule electronic properties are evaluated at the B3LYP/6-311g(d) level of theory ${ }^{60,61}$ using the NWChem code. ${ }^{62}$

All systems under study can be described by periodic primitive cells of either tetragonal or orthorhombic symmetry. In order to maintain a consistent labeling of high symmetry $k$-space pathways, the orthorhombic Brillouin zone special points $\Gamma(0,0,0), \mathrm{Y}(0,0.5$, $0)$, $S(0.5,0.5,0)$, and $X(0.5,0,0)$ are adopted for both Bravais lattices, meaning that the typical " $\Gamma-\mathrm{X}-\mathrm{M}-\Gamma$ " pathway of the tetragonal lattice would be " $\Gamma-Y-S-\Gamma$."

Charge carrier mobility estimates are drawn using the BTE theory, according to which the charge mobility of a $2 \mathrm{D}$ system can be expressed as

$$
\mu=\frac{2 e \hbar^{3} C_{2 \mathrm{D}}}{3 k_{\mathrm{B}} T\left(m^{*} E_{1}\right)^{2}},
$$

where $e$ is the elementary charge, $\hbar$ is the reduced Planck constant, $k_{\mathrm{B}}$ is the Boltzmann constant, $T$ is the temperature of the system, $m^{*}$ is the effective mass of the carrier, $C_{2 \mathrm{D}}$ is the in-plane stiffness, and $E_{1}$ is the Bardeen-Shockley deformation potential (DP). ${ }^{40,44,63-65}$

Hole and electron effective masses are calculated via quadratic fitting of the valence band (VB) and conduction band (CB) extrema, respectively. Although the utilization of the PBE XC functional is known to underestimate the electronic bandgap, ${ }^{66}$ the morphology of the band structure is consistent with results from hybrid XC functionals. ${ }^{40,44}$ As a result, all $m^{*}$ values are evaluated using PBE calculations. Moreover, both $C_{2 \mathrm{D}}$ and $E_{1}$ are calculated at the PBE level of theory by means of elastic uniaxial deformations $( \pm 0.5 \%$ and $\pm 1.0 \%$ strain) along the in-plane lattice vector directions.

\section{RESULTS AND DISCUSSION}

\section{A. Diacetylene-linked COFs}

The diacetylene-linked ultrathin COFs under consideration are illustrated in Fig. 2, along with VB maximum and CB minimum isosurfaces, as quantified by the so-called highest occupied crystal orbital (HOCO) and lowest unoccupied crystal orbital (LUCO), respectively. All crystal orbital isosurfaces presented in this work correspond to the value of $0.007 \mathrm{e} / \AA^{3}$.

The $\mathrm{P}-\mathrm{Ac} 2$ monolayer COF is a narrow bandgap 2D semiconductor, with a tetragonal in-plane lattice constant of $13.54 \AA$ and a direct PBE bandgap of $0.16 \mathrm{eV}$ at $\mathrm{S}$, further refined to $0.34 \mathrm{eV}$ via HSE06 calculations. Due to the $D_{2 h}$ point group of the metal-free porphyrin core, a degree of anisotropy to the electronic properties is to be expected. In order to quantify such anisotropies, band structure calculations are carried out using a uniform $k$-space grid on the $k_{x}-k_{y}$ plane, prompting this way the derivation of $2 \mathrm{D}$ band structure contour plots, which contain the complete symmetry of the first Brillouin zone. Once the positions of the VB and CB extrema are resolved, effective mass $m^{*}$ values can be computed by fitting a quadratic expression to the band energy along specified directions in the reciprocal space. A polar $k$-space sampling, with the polar vector centered on the band extremum while scanning the reciprocal $k_{x}-k_{y}$ plane in an anti-clockwise fashion with respect to the $(0.5,0,0)$ direction, enables capturing any anisotropic effects on the effective mass of charge carriers.

In the case of $\mathrm{P}-\mathrm{Ac} 2$, the minimum $m^{*}$ values of $0.04 \mathrm{~m}_{0}$ and $0.05 m_{0}$ for holes and electrons, respectively, correspond to the S- $\Gamma$ direction along the positive major diagonal. These values, combined with an in-plane stiffness of $53.0 \mathrm{~N} / \mathrm{m}$ and DP values of $-4.8 \mathrm{eV}$ and $-3.1 \mathrm{eV}$, yield charge carrier room temperature BTE mobilities of $20 \times 10^{3} \mathrm{~cm}^{2} / \mathrm{V} \mathrm{s}$ for holes and $31 \times 10^{3} \mathrm{~cm}^{2} / \mathrm{V}$ s for electrons. Due to the tendency of the BTE theory to overestimate charge mobilities, ${ }^{41}$ these values should be interpreted with caution and be considered as an upper estimate of CT capabilities. The band structure diagram

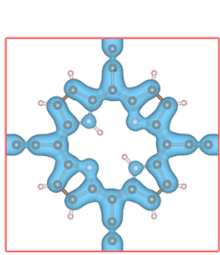

(a)

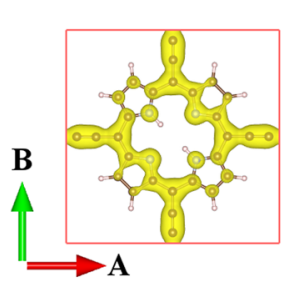

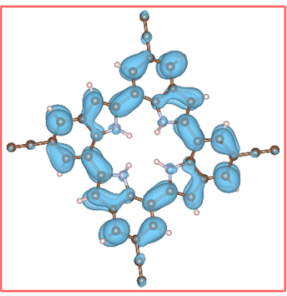

(b)

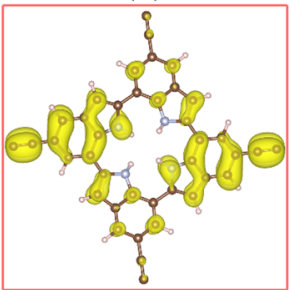

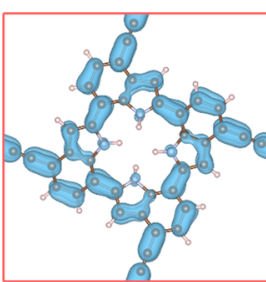

(c)

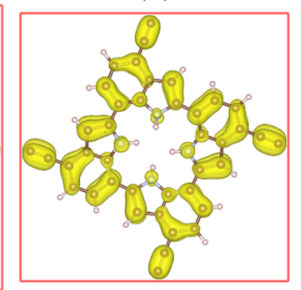

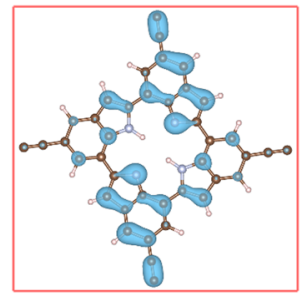

(d)

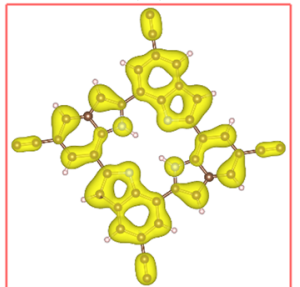

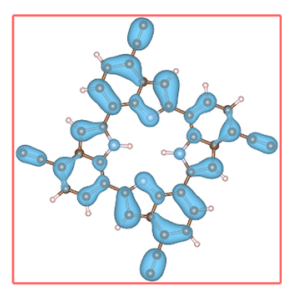

(e)

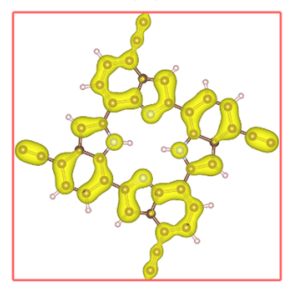

FIG. 2. HOCO (bottom panel) and LUCO (top panel) isosurfaces for diacetylene-linked COFs: (a) P-Ac2, (b) TIP-Ac2, (c) TIP(4)-Ac2, (d) TIP-2H-Ac2, and (e) TIP(4)-2HAc2. The vectors at the bottom left corner correspond to the in-plane crystallographic axes. 

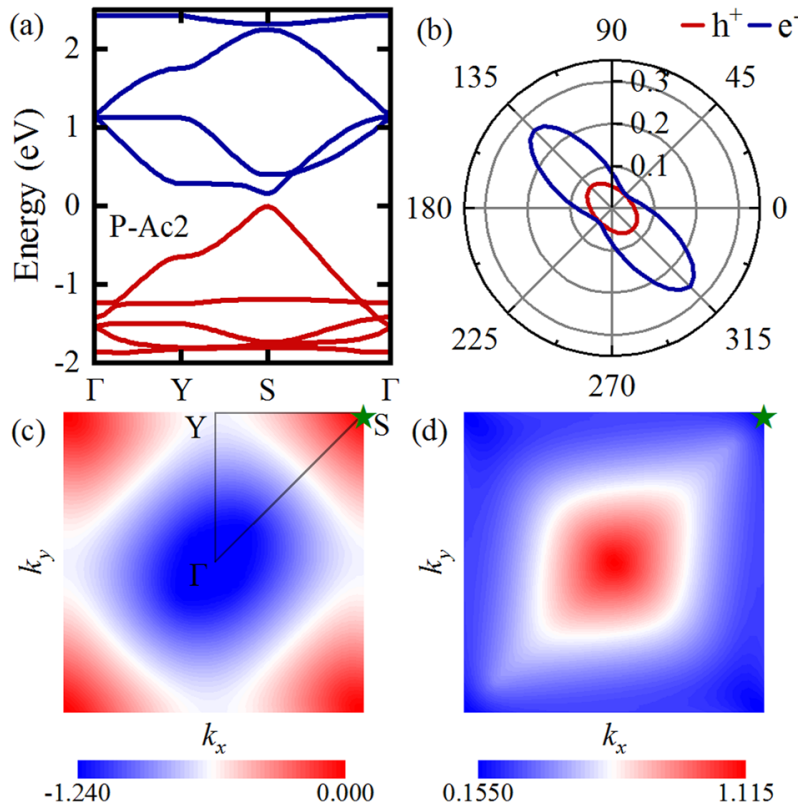

FIG. 3. (a) The band structure of P-Ac2 along high-symmetry pathways of the tetragonal unit cell. (b) Hole and electron effective mass polar diagrams. (c) Valence and (d) conduction band contour plots. The star symbol signifies band extrema.

together with the $m^{*}$ polar plot and $\mathrm{VB} / \mathrm{CB}$ contour plots for $\mathrm{P}-\mathrm{Ac} 2$ are illustrated in Fig. 3.

The replacement of the porphyrin core with tetra-indole porphyrinoid variants carries a drastic effect on the band structure of monolayer COFs. Upon introducing a TIP core to the diacetylenelinked network, the bandgap adopts an indirect $S-\Gamma$ character and is widened to $2.02 \mathrm{eV}$ (2.71 HSE06), while $\mathrm{VB}$ and $\mathrm{CB}$ dispersion is also affected, leading to more flattened bands that-in turn-augment the $m^{*}$ values by two orders of magnitude. The morphology of the frontier bands is also altered, as shown in Fig. 4.

An interesting alteration on the electronic properties takes place when changing the position of the Ac2 grafting sites, i.e., switching from the TIP to the TIP(4) core. The indirect bandgap becomes $0.92 \mathrm{eV}$ (1.37 HSE06), and the VB and CB morphology appears more symmetrical, with $m^{*}$ values of $0.3 m_{0}$ and $0.2 m_{0}$ for holes and electrons, respectively. These differences are a profound example of how the mere differentiation of grafting positions can affect properties of the periodic monolayer.

The elastic response is also affected, with the spatially averaged in-plane stiffness values being equal to $33 \mathrm{~N} / \mathrm{m}$ and $14 \mathrm{~N} / \mathrm{m}$ for the TIP-Ac2 and TIP(4)-Ac2 systems, respectively. All calculated properties for tetra-indole based diacetylene COFs are summarized in Tables I and II.

As regards the stability of diacetylene-linked porphyrin and porphyrinoid COFs, the calculated cohesive energies listed in Table I are indicative of fair structural robustness, with values ranging from $-6.89 \mathrm{eV}$ to $-7.03 \mathrm{eV}$. Their thermodynamic stability is further examined by means of Born-Oppenheimer molecular dynamics (BOMD) simulations at room temperature, employing a time step of $1 \mathrm{fs}$ over a total duration of $5 \mathrm{ps}$, with all structures remaining intact. The time evolution of the root-mean-square deviation (RMSD) of atomic positions is illustrated in Fig. 5, along with characteristic snapshots capturing the effect of functionalization positions on molecular motion. The utilization of the 4 th indole position leads to pronounced out-of-plane motion as is quantified by larger RMSD values.
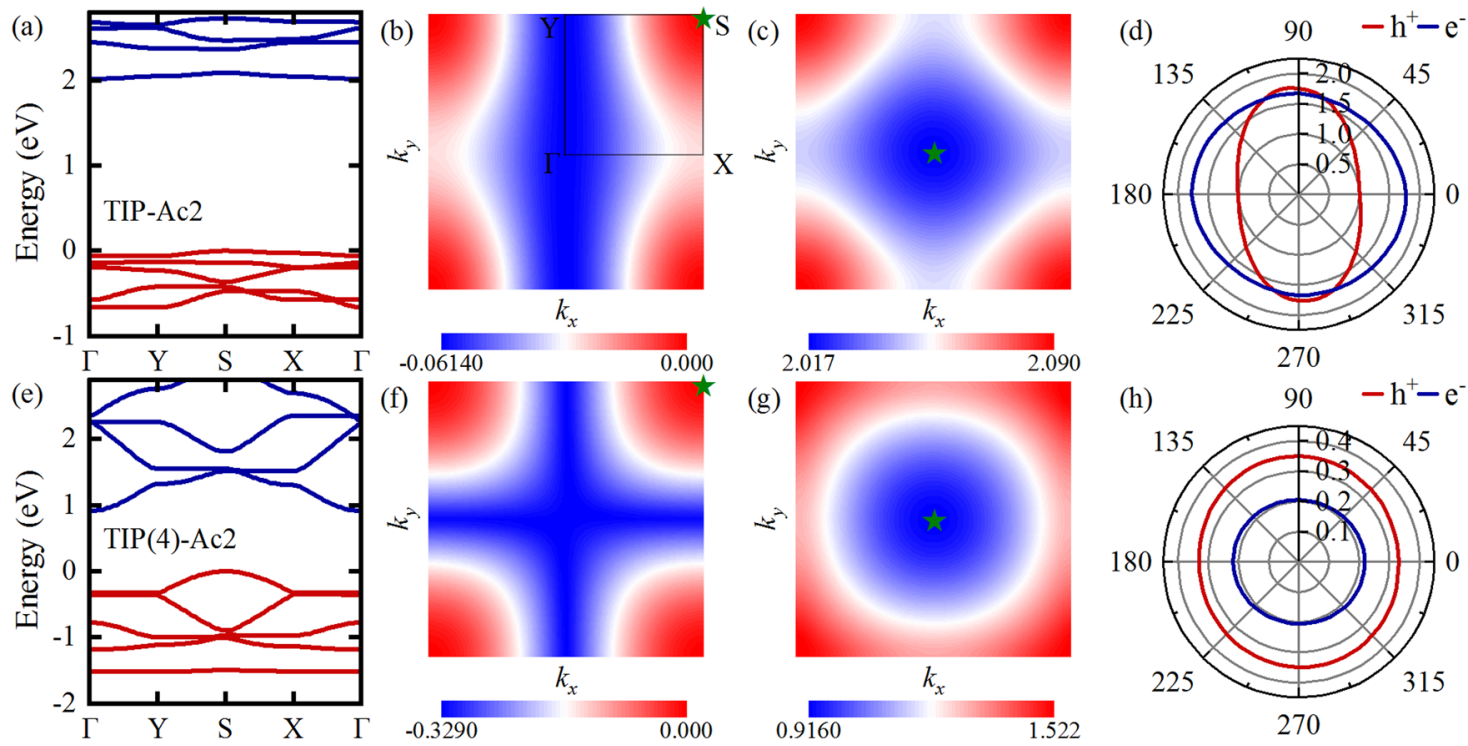

FIG. 4. Band structure diagrams (a) and (e) for diacetylene-linked TIP COFs, along with valence band (b) and (f) and conduction band (c) and ( $g$ ) contour plots, and (d) and (h) hole and electron effective mass polar diagrams. The top and bottom panels correspond to TIP cores grafted at the 5 and 4 indole positions, respectively. 
TABLE I. Tetragonal lattice parameters A, cohesive energy values, PBE electronic energy gaps (HSE06 values are listed inside parentheses), and hole $\left(\mathrm{h}^{+}\right)$and electron $\left(\mathrm{e}^{-}\right)$effective mass values, with the reciprocal space pathway specified inside parentheses, for all diacetylene-linked COFs under study.

\begin{tabular}{lccccc}
\hline \hline System & $A(\AA)$ & $E_{\text {coh }}(\mathrm{eV})$ & Energy gap $(\mathrm{eV})$ & $\mathrm{h}^{+} m^{*}\left(m_{0}\right)$ & $\mathrm{e}^{-} m^{*}\left(m_{0}\right)$ \\
\hline P-Ac2 & 13.54 & -6.97 & $0.16(0.34)^{\mathrm{a}}$ & $0.04(\mathrm{~S}-\Gamma)$ & $0.05(\mathrm{~S}-\Gamma)$ \\
TIP-Ac2 & 18.11 & -6.89 & $2.02(2.71)^{\mathrm{b}}$ & $1.01(\mathrm{~S}-\mathrm{Y})$ & $1.63(\Gamma-\mathrm{S})$ \\
TIP(4)-Ac2 & 17.01 & -6.89 & $0.92(1.37)^{\mathrm{b}}$ & $0.33(\mathrm{~S}-\mathrm{Y})$ & $0.20(\Gamma-\mathrm{X})$ \\
TIP-2H-Ac2 & 18.08 & -7.01 & $0.01(0.09)^{\mathrm{a}}$ & $0.56(\mathrm{X}-\mathrm{S})$ & $0.21(\mathrm{X}-\mathrm{S})$ \\
TIP(4)-2H-Ac2 & 17.08 & -7.03 & $0.05(0.09)^{\mathrm{a}}$ & $0.05(\mathrm{Y}-\mathrm{S})$ & $0.06(\mathrm{Y}-\mathrm{S})$ \\
\hline \hline
\end{tabular}

${ }^{\mathrm{a}}$ Direct gap.

${ }^{\mathrm{b}}$ Indirect gap.

TABLE II. In-plane stiffness and deformation potential values for diacetylene-linked COFs along the $A$ direction (values inside parentheses correspond to the $B$ direction), along with BTE lower estimates for charge carrier mobilities.

\begin{tabular}{lccccc}
\hline \hline System & & & $\begin{array}{c}\mathrm{h}^{+} \mu \\
\left(\times 10^{3} \mathrm{~cm}^{2} / \mathrm{V} \mathrm{s}\right)\end{array}$ & $\begin{array}{c}\mathrm{e}^{-} \mu \\
\left(\times 10^{3} \mathrm{~cm}^{2} / \mathrm{V} \mathrm{s}\right)\end{array}$ \\
\hline P-Ac2 & $53.0(53.0)$ & $-4.8(-4.8)$ & $-3.1(-3.1)$ & 20.41 & 31.32 \\
TIP-Ac2 & $35.3(31.4)$ & $-2.5(-2.1)$ & $-1.9(-2.3)$ & 0.08 & 0.04 \\
TIP(4)-Ac2 & $14.9(14.2)$ & $-1.3(-1.3)$ & $-1.3(-1.7)$ & 1.10 & 1.83 \\
TIP-2H-Ac2 & $40.2(39.5)$ & $-1.9(-1.3)$ & $-1.2(-2.7)$ & 0.50 & 1.78 \\
TIP(4)-2H-Ac2 & $24.1(24.4)$ & $-2.4(0.2)$ & $-0.8(-1.2)$ & 23.76 & 66.01 \\
\hline \hline
\end{tabular}

Moreover, the chemical bonding nature for all diacetylenelinked COFs under study is examined via electron localization function (ELF) calculations. Atomic bonds appear to have a strict covalent character, as is illustrated in the contour plots and $\mathrm{ELF}=0.8$ isosurfaces in Fig. 6 for three characteristic systems, where no polarization is evident for high loneliness ELF contributions in the bond domains.

The introduction of oxidized TIP cores, i.e., TIP-2H and TIP(4)-2H, leads to the manifestation of an intriguing phenomenon: the diminishing of the number of electrons by two reduces the

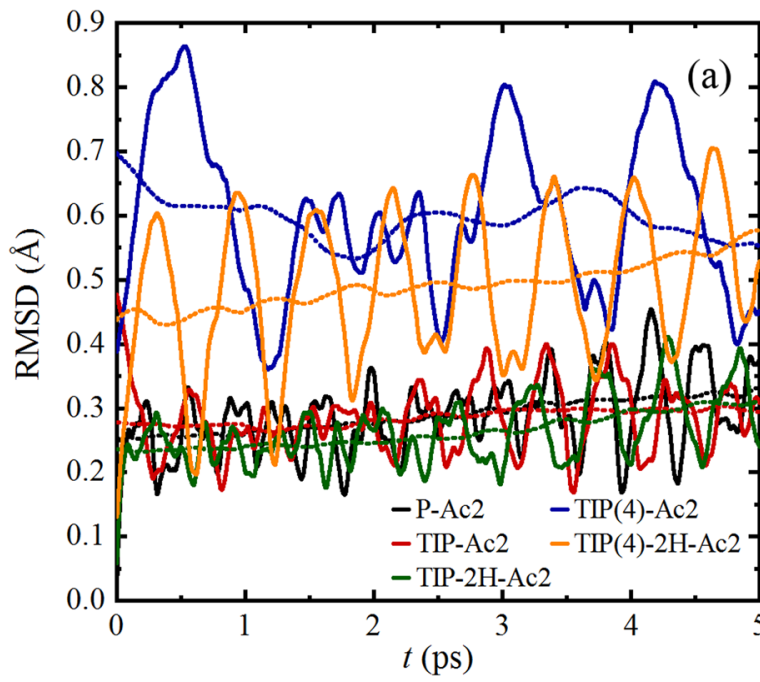

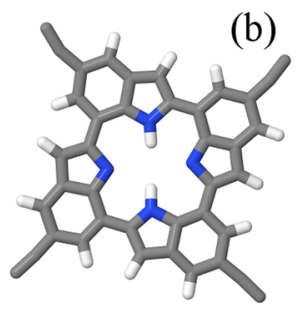

(c)

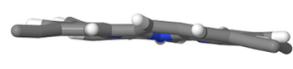

TIP-2H-Ac2

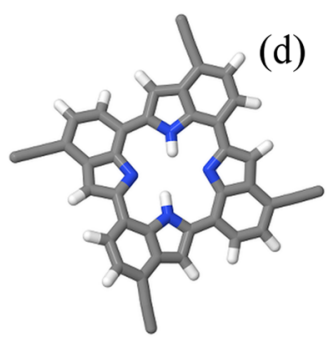

(e)

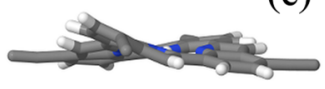

TIP(4)-2H-Ac2

FIG. 5. (a) Room temperature RMSD time series for diacetylene-linked ultrathin COFs. The dotted curves correspond to the moving average. The right panel illustrates top and side views of TIP-2H-Ac2 (b) and (c) and TIP(4)-2H-Ac2 (d) and (e) molecular snapshots at 5 ps. 

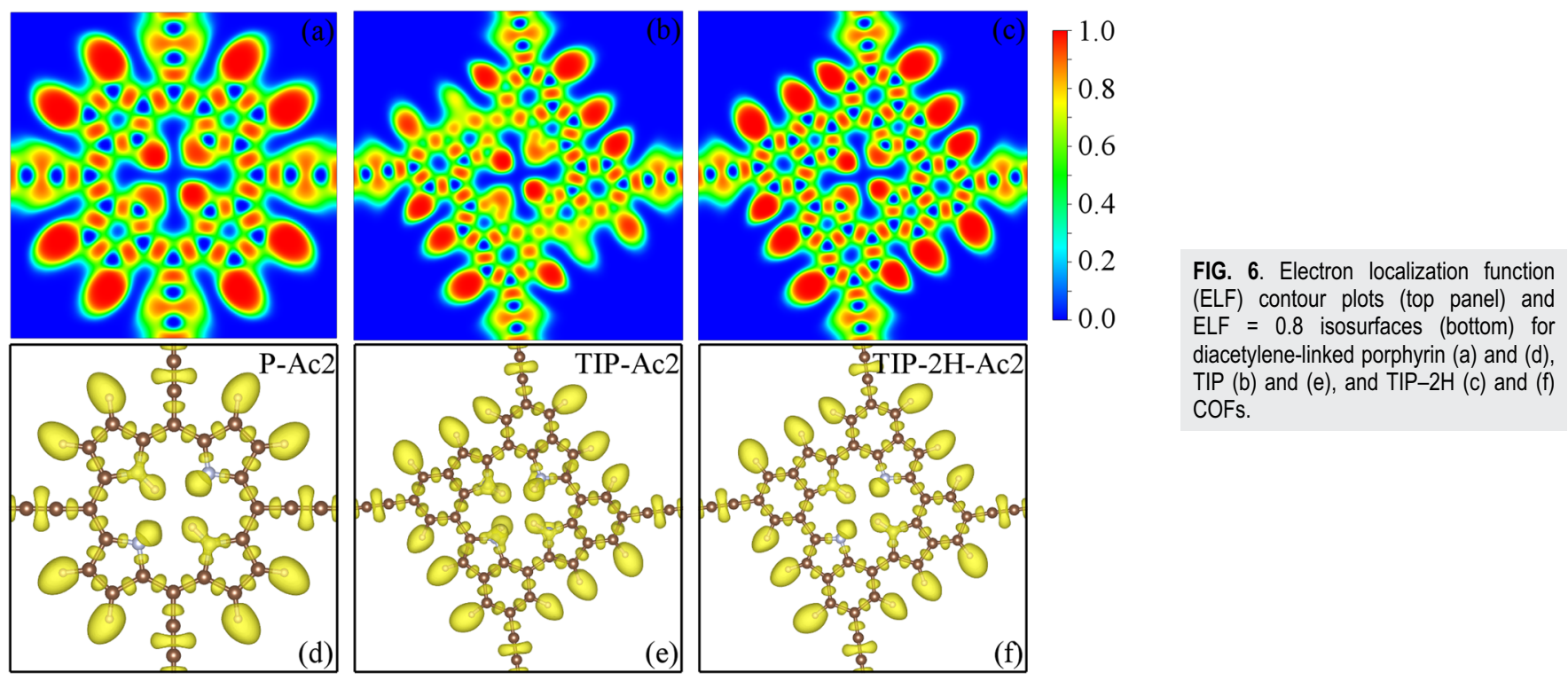

HSE06 bandgap to $0.1 \mathrm{eV}$, while simultaneously inducing significant anisotropic effects on the electronic band landscape, as is clearly demonstrated in Fig. 7.

The $m^{*}$ anisotropy is a consequence of the altered band occupation compared to fully hydrogenated TIP cores, forcing charge carriers to occupy crystal orbitals with an extended flat band character in the reciprocal space. The differences in the mechanical properties regarding the $C_{2 \mathrm{D}}$ values follow the same trend with the TIP-based COFs. The most noticeable variation in the electronic response to external strain is for the TIP(4)-2H-Ac2 system, with a sign reversal for hole DP values and an order of magnitude difference to the absolute value when applying deformations along the different crystallographic directions.
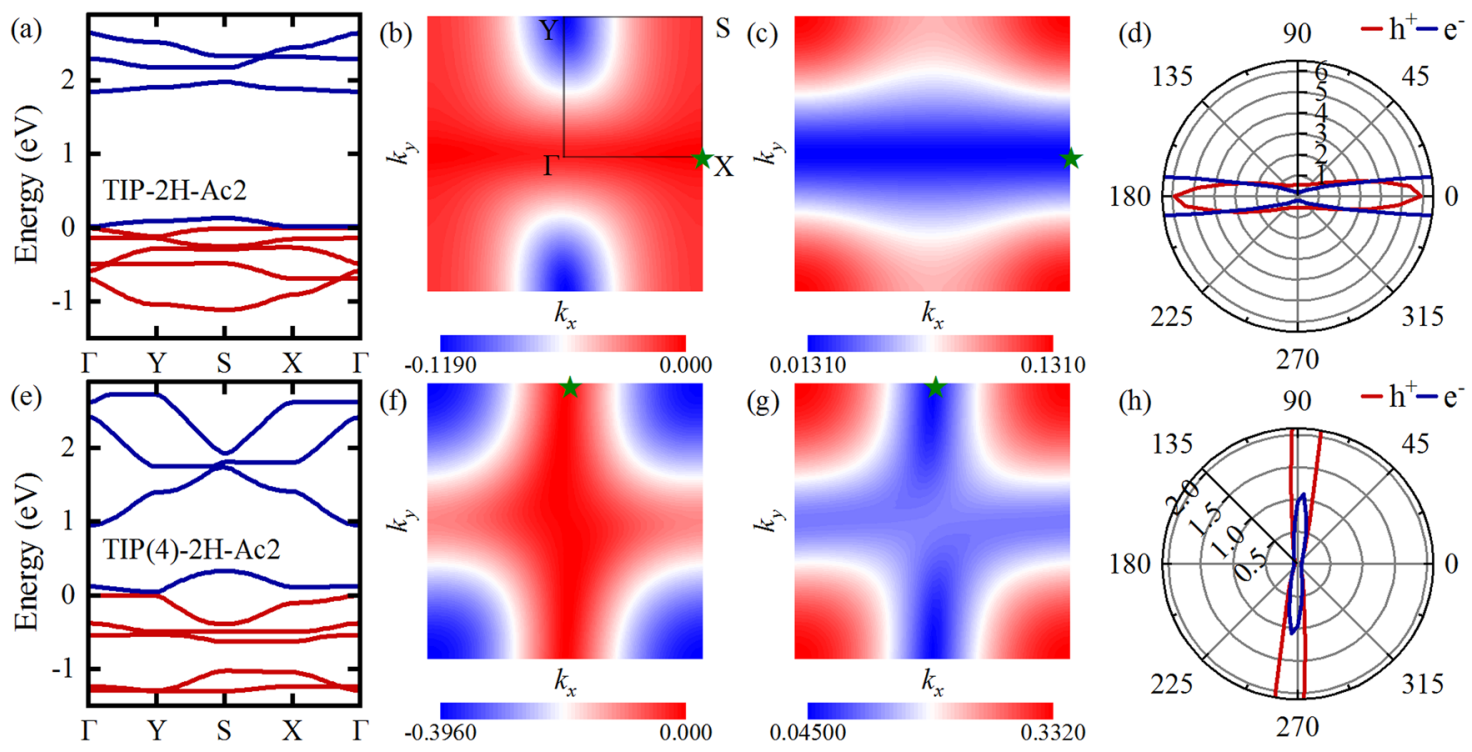

FIG. 7. Band structure diagrams (a) and (e) for diacetylene-linked TIP-2H COFs, along with valence band (b) and (f) and conduction band (c) and (g) contour plots, and (d) and $(h)$ hole and electron effective mass polar diagrams. The top and bottom panels correspond to TIP-2H cores grafted at the 5 and 4 indole positions, respectively. 
TABLE III. Cohesive energy values $E_{\text {coh }}$ (expressed in $\mathrm{eV}$ ) and equilibrium atomic RMSD values by means of room temperature BOMD simulations (expressed in $\AA$ ) for phenyl, pyrene, diphenyl-diacetylene, and TBAA linked porphyrin and tetra-indole COFs.

\begin{tabular}{|c|c|c|c|c|c|c|c|c|}
\hline & \multicolumn{2}{|c|}{$\mathrm{Ph}$} & \multicolumn{2}{|c|}{ Py } & \multicolumn{2}{|c|}{$\mathrm{Ph} 2 \mathrm{Ac} 2$} & \multicolumn{2}{|c|}{ TBAA } \\
\hline & $E_{\mathrm{coh}}$ & RMSD & $E_{\mathrm{coh}}$ & RMSD & $E_{\mathrm{coh}}$ & RMSD & $E_{\mathrm{coh}}$ & RMSD \\
\hline $\mathrm{P}$ & -6.55 & 0.3 & -6.70 & 0.2 & -6.60 & 0.6 & -6.39 & 0.8 \\
\hline TIP & -6.60 & 0.5 & -6.71 & 0.7 & -6.63 & 0.6 & -6.44 & 1.1 \\
\hline $\mathrm{TIP}(4)$ & -6.60 & 0.5 & -6.71 & 0.7 & -6.63 & 0.7 & -6.44 & 0.8 \\
\hline TIP-2H & -6.69 & 0.7 & -6.77 & 0.2 & -6.69 & 0.4 & -6.49 & 0.8 \\
\hline TIP(4)-2H & -6.70 & 0.6 & -6.68 & 0.8 & -6.70 & 0.5 & -6.49 & 0.7 \\
\hline
\end{tabular}

The pronounced variations in electronic properties upon the introduction of oxidized TIP-2H cores are attributed to the fundamental differences in a single molecule electronic structure of porphyrin, TIP, and TIP- $2 \mathrm{H}$ cores. The isolated metal-free porphyrin molecule has a highest occupied molecular orbital (HOMO) and lowest unoccupied molecular orbital (LUMO) gap of $2.92 \mathrm{eV}$, while the HOMO-LUMO gaps of TIP and TIP-2H molecules are $3.43 \mathrm{eV}$ and $0.62 \mathrm{eV}$, respectively, hence the drastic diminishing of the bandgap when TIP- $2 \mathrm{H}$ cores are used for the formation of the COF monolayer.

In general, the HOMO-LUMO gap of an isolated molecule is dictated by the molecular morphology and chemical composition. In the case of polyaromatic systems, the gap value is inversely proportional to the conjugation extension. ${ }^{67}$ As a result, oxidized TIP-2H cores have a lower gap compared to porphyrin since the $\pi$-conjugation between coplanarly linked indoles in the TIP-2H molecule is more pronounced than $s p^{2}$ carbon linked pyrroles in porphyrins. Such a behavior has also been demonstrated in the literature, e.g., in the work of Saegusa et al. on ring-fused porphyrins. ${ }^{68}$ On the other hand, the reduced TIP core has a diminished aromatic character, thus raising the gap value. Upon core polymerization via the mediation of appropriate spacers, the conjugation extension leads to a further decrease in the electronic bandgap, similarly to the $N$-band formation in polyenes. ${ }^{69}$

The bandgap of TIP-based systems can-in principle-be further tuned via metal complexation: the introduction of $\mathrm{Ni}$ or $\mathrm{Zn}$ atoms in the TIP core brings the HOMO-LUMO single molecule gaps to $0.60 \mathrm{eV}$ and $0.70 \mathrm{eV}$, respectively, whereas the utilization of $\mathrm{Co}$ or $\mathrm{Cu}$ leads to open-shell cores with gap values of $0.76 \mathrm{eV}$ and $0.96 \mathrm{eV}$. As a result, metal-containing TIP COFs could form a new, versatile, and tunable family of narrow bandgap 2D semiconductors.

\section{B. Phenyl, pyrene, diphenyl-diacetylene, and TBAA linked COFs}

Different combinations of porphyrin and tetra-indole cores and twofold articulated linkers can form stable 2D ultrathin compounds, as is corroborated by the cohesive energy and room temperature atomic RMSD equilibrium values listed in Table III. Such systems are found to exhibit variable electronic properties with respect to the band structure and the charge carrier localization profile.
As regards the porphyrin-based monolayers under study, charge localization, as quantified by HOCO and LUCO isosurfaces, is modulated by the type of the linker. The most noticeable disruption of carrier delocalization occurs in the P-Py COF, with the majority of the density accumulated onto the porphyrin core due to the large core-linker torsional angle of $64^{\circ}$, which disrupts the $\pi$ resonance across the $2 \mathrm{D}$ network $(\mathrm{P}-\mathrm{Ph}$ and $\mathrm{P}-\mathrm{Ph} 2 \mathrm{Ac} 2$ exhibit torsional angles of $57^{\circ}$ and $62^{\circ}$, respectively). Selected isosurfaces are shown in Fig. 8.

Moreover, the utilization of the nitrogen-containing TBAA linker leads to a mild HOCO and LUCO spatial separation, suggesting that potentially photogenerated hole and electron charge
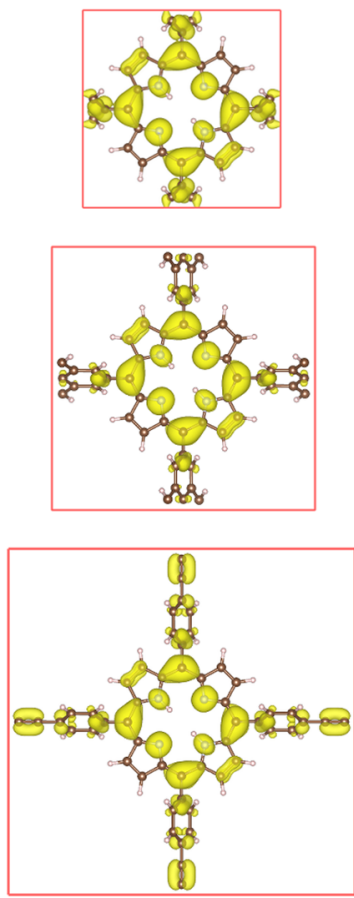

(a)

\section{(b)}

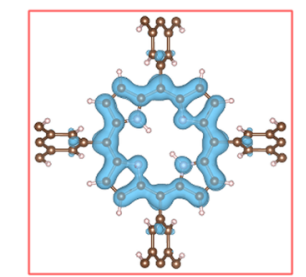

(c)

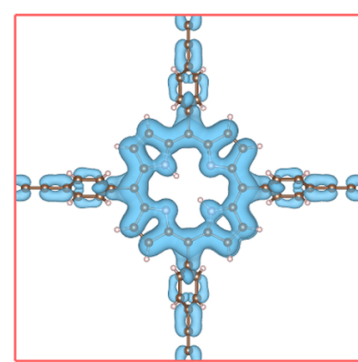

FIG. 8. HOCO (left panel) and LUCO (right panel) isosurfaces for porphyrin-based COFs with variable linkers: (a) Ph, (b) Py, and (c) Ph2Ac2. 
TABLE IV. Lattice parameter $A$ and PBE energy gap values (HSE06 values in parentheses) for phenyl, pyrene, diphenyldiacetylene, and TBAA linked porphyrin and tetra-indole COFs.

\begin{tabular}{|c|c|c|c|c|c|c|c|c|}
\hline & \multicolumn{2}{|r|}{$\mathrm{Ph}$} & \multicolumn{2}{|c|}{ Py } & \multicolumn{2}{|c|}{$\mathrm{Ph} 2 \mathrm{Ac} 2$} & \multicolumn{2}{|c|}{ TBAA } \\
\hline & $A(\AA)$ & Gap (eV) & $A(\AA)$ & Gap (eV) & $A(\AA)$ & Gap (eV) & $A(\AA)$ & Gap (eV) \\
\hline $\mathrm{P}$ & 12.79 & $1.33(1.75)$ & 17.02 & 1.57 & 22.23 & 1.46 & 25.60 & 1.38 \\
\hline TIP & 17.26 & $2.16(2.86)$ & 21.45 & 2.16 & 26.61 & 2.08 & 29.81 & 1.64 \\
\hline $\mathrm{TIP}(4)$ & 16.30 & $1.59(2.13)$ & 20.30 & 1.72 & 25.34 & 1.49 & 29.07 & 1.30 \\
\hline TIP-2H & 17.26 & $0.14(0.28)$ & 21.46 & 0.17 & 26.63 & 0.12 & 29.81 & 0.17 \\
\hline TIP(4)-2H & 16.39 & $0.12(0.13)$ & 21.46 & 0.06 & 25.51 & 0.04 & 29.24 & 0.14 \\
\hline
\end{tabular}

TABLE V. Hole and electron effective mass values (expressed in $m_{0}$ ) for phenyl, pyrene, diphenyl-diacetylene, and TBAA linked porphyrin and porphyrinoid tetra-indole COFs. Superscripts indicate different reciprocal space pathways.

\begin{tabular}{|c|c|c|c|c|c|c|c|c|}
\hline & \multicolumn{2}{|c|}{$\mathrm{Ph}$} & \multicolumn{2}{|c|}{ Py } & \multicolumn{2}{|c|}{$\mathrm{Ph} 2 \mathrm{Ac} 2$} & \multicolumn{2}{|c|}{ TBAA } \\
\hline & $\mathrm{h}^{+} m^{*}$ & $\mathrm{e}^{-} m^{*}$ & $\mathrm{~h}^{+} m^{*}$ & $\mathrm{e}^{-} m^{*}$ & $\mathrm{~h}^{+} m^{*}$ & $\mathrm{e}^{-} m^{*}$ & $\mathrm{~h}^{+} m^{*}$ & $\mathrm{e}^{-} m^{*}$ \\
\hline$P$ & $0.50^{\mathrm{a}, \mathrm{b}}$ & $0.60,{ }^{\mathrm{a}} 0.77^{\mathrm{b}}$ & $0.72,{ }^{\mathrm{a}} 0.81^{\mathrm{b}}$ & $0.76,{ }^{\mathrm{a}} 0.81^{\mathrm{b}}$ & $0.40,{ }^{\mathrm{a}} 0.42^{\mathrm{b}}$ & $0.47,{ }^{\mathrm{a}} 0.54^{\mathrm{b}}$ & $1.15,{ }^{\mathrm{a}} 0.96^{\mathrm{b}}$ & $0.72,{ }^{\mathrm{a}} 0.96^{\mathrm{b}}$ \\
\hline TIP & $12.57^{\mathrm{a}}, 6.31^{\mathrm{b}}$ & $3.16^{\mathrm{c}, \mathrm{d}}$ & $8.18,{ }^{\mathrm{a}} 8.17^{\mathrm{b}}$ & $2.04,{ }^{c} 4.10^{d}$ & $1.76,{ }^{\mathrm{a}} 1.33^{\mathrm{b}}$ & $0.89,^{\mathrm{c}} 1.06^{\mathrm{d}}$ & $4.22,{ }^{\mathrm{a}} 2.12^{\mathrm{b}}$ & $\infty^{c, d}$ \\
\hline $\operatorname{TIP}(4)$ & $0.59^{\mathrm{a}, \mathrm{b}}$ & $0.39,{ }^{c} 0.41^{\mathrm{d}}$ & $0.61,{ }^{\mathrm{a}} 0.57^{\mathrm{b}}$ & $0.51,{ }^{\mathrm{c}} 0.44^{\mathrm{d}}$ & $0.42^{\mathrm{a}, \mathrm{b}}$ & $0.29,{ }^{c} 0.33^{d}$ & $0.63,{ }^{\mathrm{a}} 1.11^{\mathrm{b}}$ & $0.74,{ }^{\mathrm{c}} 0.56^{\mathrm{d}}$ \\
\hline TIP-2H & $1.20,{ }^{\mathrm{a}} 0.97^{\mathrm{b}}$ & $4.20,{ }^{c} 6.32^{\mathrm{d}}$ & $8.16,{ }^{\mathrm{c}} 1.82^{\mathrm{d}}$ & $\infty,{ }^{c} 5.45^{\mathrm{d}}$ & $0.53,{ }^{c} 0.89^{d}$ & $1.33,{ }^{\mathrm{c}} 2.66^{\mathrm{d}}$ & $1.06,{ }^{\mathrm{c}} 2.12^{\mathrm{d}}$ & $4.23,{ }^{c} 8.49^{d}$ \\
\hline $\mathrm{TIP}(4)-2 \mathrm{H}$ & $0.16^{\mathrm{e}}$ & $0.50,{ }^{c} 0.52^{d}$ & $0.27^{\mathrm{e}}$ & $0.54,{ }^{c} 0.39^{d}$ & $0.10,{ }^{c} 0.22^{d}$ & $0.12,{ }^{\mathrm{c}} 0.17^{\mathrm{d}}$ & $0.40,{ }^{c} 0.74$ & $0.49,{ }^{c} 0.63^{\mathrm{d}}$ \\
\hline
\end{tabular}

${ }^{\mathrm{a}} \mathrm{S}-\mathrm{G}$.

${ }^{\mathrm{b}} \mathrm{S}-\mathrm{Y}$.

${ }^{\mathrm{c}} \mathrm{G}-\mathrm{Y}$.

${ }^{\mathrm{d}} \mathrm{G}-\mathrm{S}$.

${ }^{\mathrm{e}} \mathrm{Y}-\mathrm{S}$.

carriers will accumulate on the porphyrin and TBAA fragments, respectively.

As far as the band structure is concerned, the direct nature of the bandgap at $\mathrm{S}$ is maintained for porphyrin-based COFs, with PBE gap values in the interval of $1.3 \mathrm{eV}$ and $1.6 \mathrm{eV}$. The band curvature is also affected by the choice of the linker, resulting in in-plane $m^{*}$ values for electrons and holes ranging from $0.4 m_{0}-1.1 m_{0}$. A detailed record of all aforementioned quantities, along with in-plane lattice constants, is reported in Tables IV and V.

Due to the computationally demanding nature of DFT calculations employing the HSE06 range-separated hybrid XC functional, its utilization for electronic structure calculations was limited for diacetylene- and phenyl-linked COFs. The calculated HSE06 bandgap values exhibit a linear scaling with respect to their PBE counterparts, hence enabling a linear fitting that enables a correction to PBE gap values for larger systems, as is illustrated in Fig. 9. The calculated and predicted HSE06 bandgaps for all systems under study are characteristic of wide bandgap semiconducting behavior, with the majority of the values concentrated in the region between $1.75 \mathrm{eV}$ and $2.3 \mathrm{eV}$.

Metal complexation can effectively be used as a mechanism for further electronic bandgap engineering. Zinc and nickel containing porphyrin $2 \mathrm{D}$ COFs with adequate core spacing, i.e., utilizing linkers larger than diacetylene, register gap values in the $1.75 \mathrm{eV}$ and

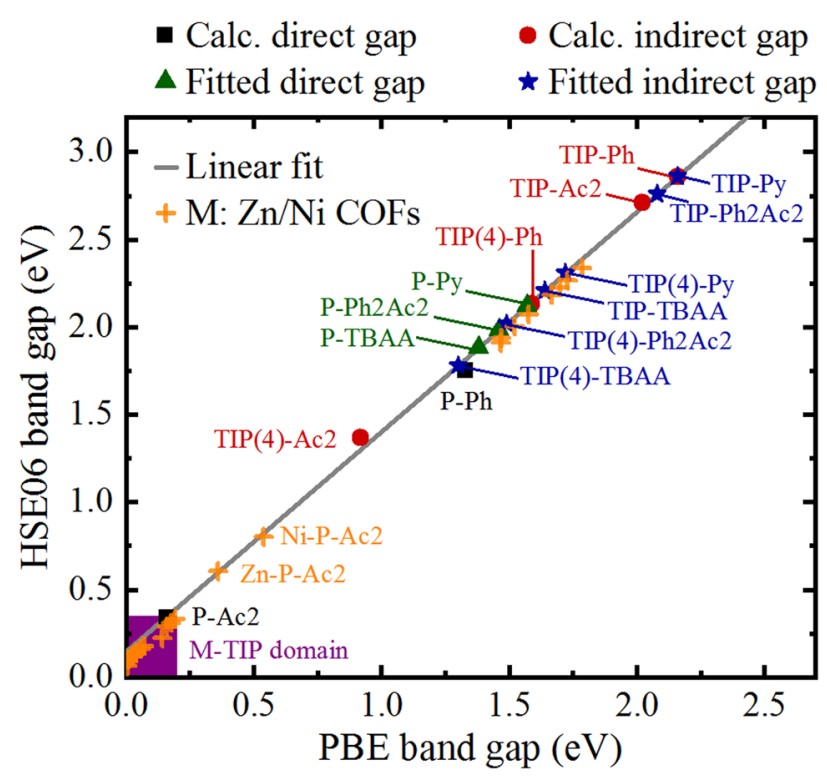

FIG. 9. PBE and HSE06 electronic bandgap values for porphyrin- and TIP-based ultrathin COFs under study. 
TABLE VI. Positive PBE energy gap values (expressed in eV; HSE06 values in parentheses) for $\mathrm{Zn}$ - and Ni-containing porphyrin and tetra-indole 2D COFs.

\begin{tabular}{lccccc}
\hline \hline & Ac2 & Ph & Py & Ph2Ac2 & TBAA \\
\hline Zn-P & $0.36(0.61)$ & $1.47(1.91)$ & 1.70 & 1.58 & 1.47 \\
Zn-TIP & $\ldots$ & $0.14(0.22)$ & 0.18 & 0.04 & 0.19 \\
Zn-TIP(4) & $\ldots$ & $0.01(0.06)$ & 0.07 & 0.06 & 0.16 \\
Ni-P & $0.54(0.80)$ & $1.66(2.18)$ & 1.79 & 1.73 & 1.52 \\
Ni-TIP & $\ldots$ & $\ldots$ & 0.02 & 0.01 & 0.04 \\
Ni-TIP(4) & $\ldots$ & $\ldots$ & $\ldots$ & 0.01 & 0.03 \\
\hline
\end{tabular}

$2.3 \mathrm{eV}$ region, in complete analogy to their metal-free counterparts. On the other hand, diacetylene-linked $\mathrm{Zn}-\mathrm{P}-\mathrm{Ac} 2$ and Ni-P-Ac2 ultrathin COFs have explicitly HSE06 calculated bandgaps of $0.61 \mathrm{eV}$ and $0.80 \mathrm{eV}$, respectively, hence partially bridging the value chasm between $0.34 \mathrm{eV}(\mathrm{P}-\mathrm{Ac2})$ and $1.37 \mathrm{eV}$ [TIP(4)-Ac2]. Finally, the insertion of closed-shell metallic species such as zinc and nickel to TIP-based COFs is found to further reduce the bandgap, creating with way a subcategory of ultrathin COFs with electronic gap values ranging from zero to $\sim 0.33 \mathrm{eV}$. All positive bandgap calculated values are listed in Table VI and are represented in Fig. 9 by cross symbols.

The electronic band structure at the PBE level of theory for all the remaining combinations of porphyrin and tetra-indole based cores combined with the articulated twofold linkers under study is elucidated in Figs. 10 and 11. The trends identified in the case of diacetylene-linked COFs are present for longer linking units as well. TIP cores with linkers grafted at the 5 indole position demonstrate flat valence and conduction bands, whereas TIP(4) cores form more dispersive valence and conduction bands, with both COF categories exhibiting an $\mathrm{S}-\Gamma$ indirect bandgap. The utilization of oxidized TIP$2 \mathrm{H}$ cores leads to a diminishing of the bandgap, with TIP $(4)-2 \mathrm{H}$
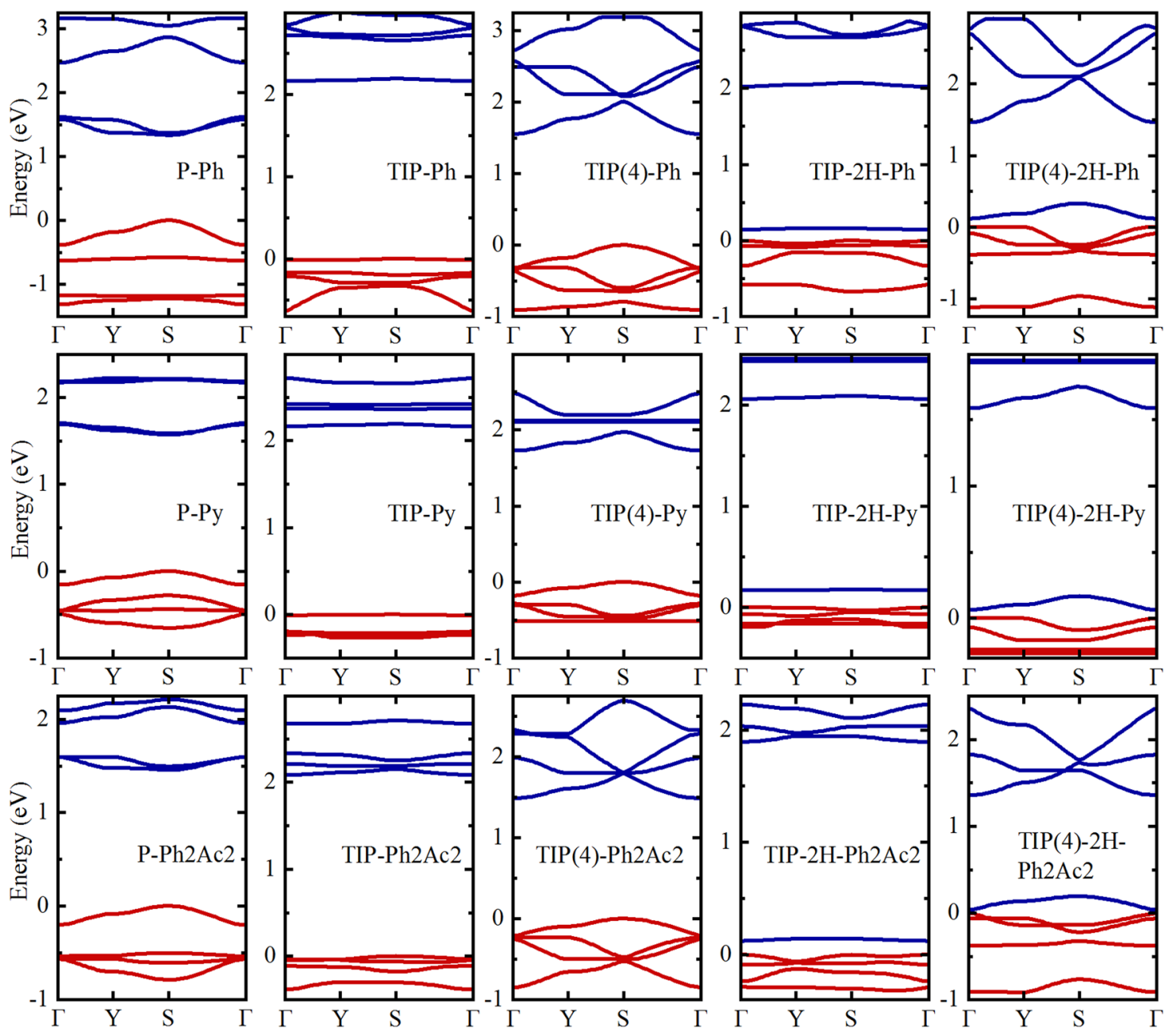

FIG. 10. Band structure diagrams of phenyl-linked (top panel), pyrene-linked (middle panel), and diphenyl-diacetylene-linked (bottom) porphyrin and tetra-indole porphyrinoid COF monolayers. 

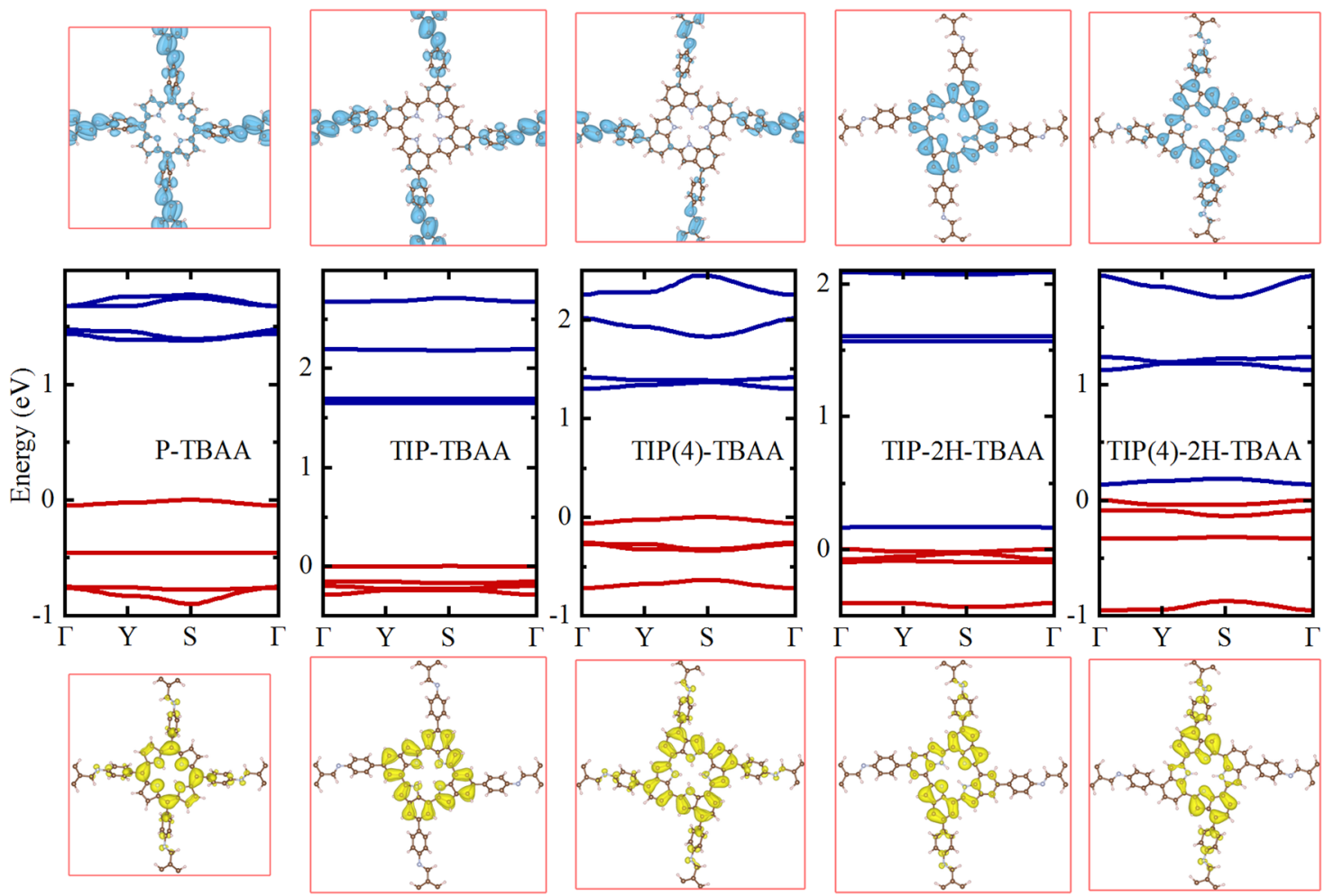

FIG. 11. Band structure diagrams of TBAA-linked porphyrin and tetra-indole porphyrinoid COF monolayers, along with HOCO (bottom panel) and LUCO (top panel) isosurfaces.

COF monolayers displaying frontier bands with more pronounced curvature compared to their TIP-2H counterparts.

Furthermore, the TIP core oxidation is found to have an effect on the localization of electron and hole charge carriers. In the case of TBAA-linked COFs, fully hydrogenated TIP cores exhibit the same spatial separation profile for the HOCO and LUCO as in the case of the P-TBAA reference system, but with a more pronounced tendency toward the depletion of the porphyrinoid core states in the case of electron localization. This behavior is totally reversed when oxidized TIP-2H cores are used for the formation of the COF monolayer, forcing both hole and electron charge carrier localization on the TIP-2H core segment of the single layer COF. Relevant crystal orbital isosurfaces accompany the band structure diagrams in Fig. 11.

\section{Fused tetra-indole semi-metallic monolayers}

Cyclic tetra-indoles can also be utilized as precursor molecules toward the formation of novel ultrathin structures without the use of articulated linkers. Direct core fusion via $s p^{2}$-hybridized carbon or nitrogen atoms can be achieved, leading to planar holey structures with extended $\pi$-conjugation.

Focusing on the oxidized TIP- $2 \mathrm{H}$, a core fusion route using carbon atom bridging on the 4 and 5 indole positions results in the
TIP-COF monolayer. This structure crystallizes in an orthorhombic primitive cell with in-plane lattice constants of $13.62 \AA$ and 13.69 $\AA$ along the typical $A$ and $B$ directions. A similar structure can be obtained via the utilization of nitrogen atom bridging on the same indole positions, leading to the TIP-N-COF $2 \mathrm{D}$ structure, also crystallizing in an orthorhombic lattice with $A=12.74 \AA$ and $B=12.76 \AA$. Both structures appear stable, with cohesive energy values of $-7.04 \mathrm{eV}$ and $-7.28 \mathrm{eV}$ for TIP-COF and TIP-N-COF, respectively.

An alternative and isoelectronic to TIP-N-COF nitrogenbridged holey structure based on the TIP-2H core can be formed via core fusion on the 5 and 6 indole positions, resulting in the TIP$\mathrm{N}-\mathrm{COF}(5,6)$ system. This structure has a slightly larger orthorhombic primitive cell with in-plane lattice constants $A=13.45 \AA$ and $B=13.54 \AA$, while appearing as the most stable TIP-2H fused system with a cohesive energy of $-7.30 \mathrm{eV}$.

The electronic DOS spectra of the three TIP-2H holey monolayers at the PBE level of theory depicted in Fig. 12 are indicative of metallic behavior.

Bearing in mind the bandgap underestimation tendency of the PBE XC functional, a further refinement of the electronic properties is carried out by means of the HSE06 functional. The calculated electronic band structure reveals that all fused TIP-2H ultrathin COFs exhibit a semi-metallic character, with a characteristic Dirac cone along the $\Gamma-S$ pathway. 

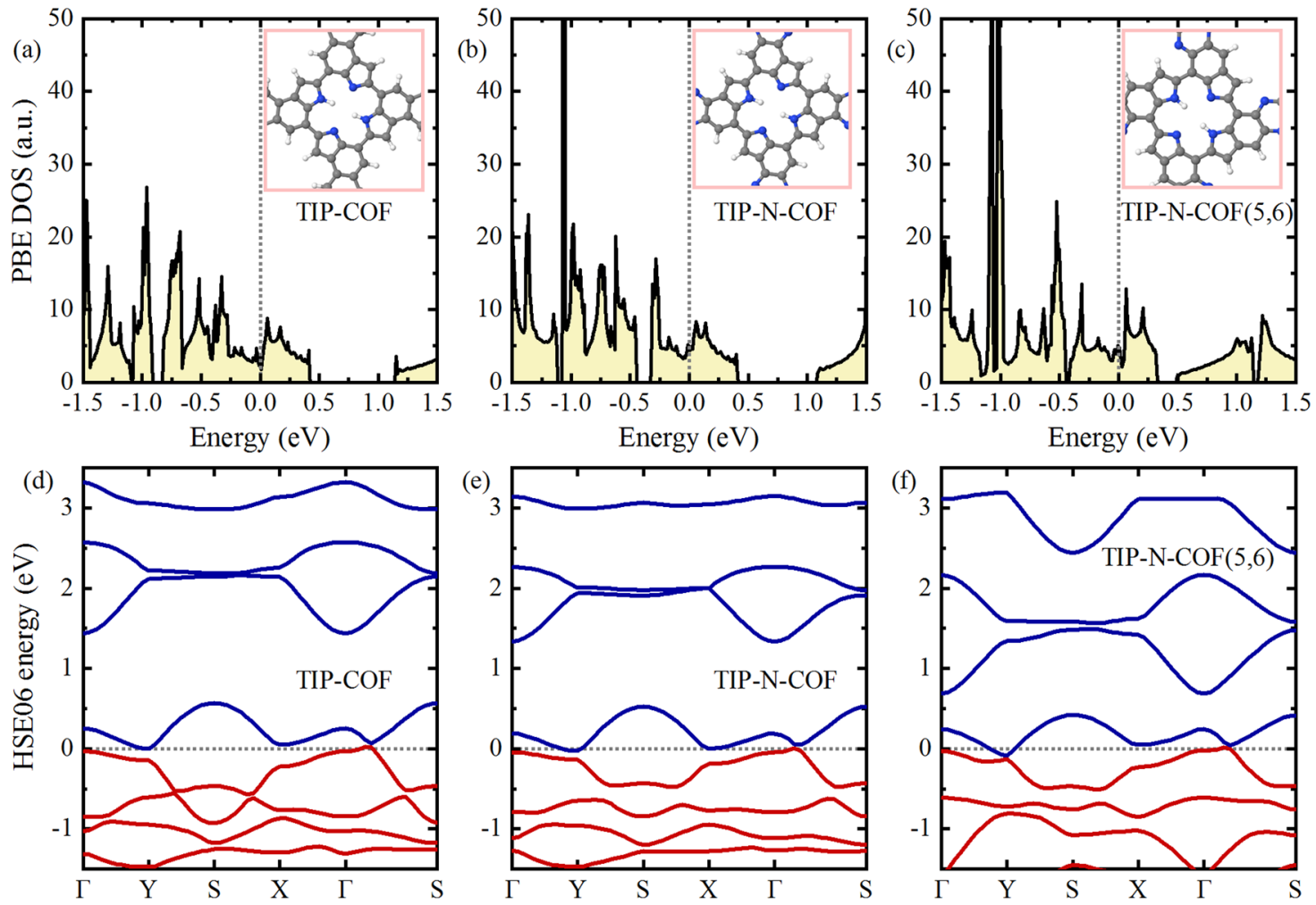

FIG. 12. (a)-(c) Electronic DOS spectra using the PBE functional and (d)-(f) band structure diagrams using the range-separated HSE06 hybrid functional along high symmetry directions of the orthorhombic primitive cell of fused TIP-2H monolayers. All energy values are scaled with respect to the Fermi level, which is highlighted by the gray dotted line.

\section{CONCLUSIONS}

The tunability of electronic and charge transport properties of fourfold porphyrin and tetra-indole porphyrinoid based COFs is demonstrated by means of DFT simulations. The core oxidation state and the grafting positions of the twofold linkers can be exploited as mechanisms toward the customization of COF properties. Furthermore, the molecular identity of articulated twofold linkers can also be considered as an additional control parameter for the tuning of the electronic band structure and charge carrier localization.

Tetra-indole porphyrinoids can be considered as promising building blocks for future 2D materials for charge transport and optoelectronic applications. We demonstrate the ability to modulate properties of ultrathin COFs via minor alterations to the chemical composition and network topology. Moreover, their full potential can be further augmented via metal complexation, as is demonstrated for zinc and nickel containing closed-shell COFs, with a tunable electronic response ranging from semi-metals to semiconductors.

Diacetylene-linked ultrathin COFs exhibit narrow bandgap semiconducting behavior with extended charge carrier delocalization. The utilization of oxidized TIP-2H cores leads to pronounced electronic band structure anisotropy due to combinations of dispersive and flat bands and results in the realization of $2 \mathrm{D}$ structures with reduced bandgap and sizable charge transport qualities when compared to the prototype diacetylene-linked porphyrin $\mathrm{COF}^{41}$

As regards the charge transport capabilities of diacetylenelinked 2D COFs under study, the semiclassical BTE computational framework returns profoundly high charge mobility values for specific systems. The utilization of this methodology is mostly justified for similar organic and inorganic materials, provided that charge carrier scattering is dominated by acoustic phonons. ${ }^{40,44,63,70-73} \mathrm{Nev}$ ertheless, all reported values should be interpreted in a comparative manner. In future work, we intend to investigate polaronic CT mechanisms inherent in ultrathin porphyrin and porphyrinoid COFs by means of mixed quantum-classical non-adiabatic molecular dynamics simulations utilizing our recently developed fragment orbital-based surface hopping methodology, ${ }^{74-78}$ hence providing a realistic and free of model assumption picture for CT phenomena for such systems.

Monolayer COFs resulting from the utilization of larger twofold linkers, such as the phenyl, pyrene, diphenyl-diacetylene, and TBAA units, demonstrate a variety of bandgap values and direct or indirect nature, primarily constituting wide bandgap 2D semiconducting materials, with electronic bandgap values and charge carrier 
effective masses comparable to other novel systems in the literature, ${ }^{40}$ thus augmenting the list of ultrathin COFs with variable pore dimensions for potential organic electronics applications.

Carrier localization is quantified in this work by means of HOCO/LUCO isosurfaces corresponding to charge density contributions of near-gap states. This ground-state DFT approach is commonly used for examining localization profiles, ${ }^{26-28,34,35}$ with fair comparison with experimental measurements. ${ }^{15}$ In the case of tetra-indole porphyrinoids, the ability to control crystal orbital localization for holes and electrons through the selection of appropriate precursor molecules could add a new family of materials for 3D stacked COF organic electronics, similar to the porphyrinand phthalocyanine-based nanostructures already registering high charge carrier mobility values. ${ }^{21,22}$

The direct fusion of tetra-indoles via $s p^{2}$-hybridized carbon and nitrogen atoms leads to the formation of holey nanostructures, with pronounced planarity and stability, while simultaneously manifesting a semi-metallic character. Such systems can complement semiconducting monolayers based on phthalocyanine derivatives ${ }^{44}$ and other similar planar nanostructures ${ }^{39}$ for the realization of hybrid materials with applications in the field of synthetic metals.

In an attempt to provide some insight toward the experimental community for the synthesis of such novel COF structures, this study puts forward some promising systems for various applications. 2D semi-metals can be formed by direct fusion of tetra-indoles, while narrow bandgap semiconducting behavior is identified in monolayer tetra-indole COFs containing closedshell metallic species such as zinc and nickel. Furthermore, carefully selected core and linker combinations yield systems with variable charge localization: oxidized TIP-2H cores with TBAA linkers have hole and electron charge carrier densities concentrated on core segments, an appealing property for directional polaronic transport in bulk, stacked systems. On the other hand, the utilization of reduced tetra-indole cores with the TBAA linker leads to a material with an electronic gap inside the visible spectrum and spatial carrier separation that can be exploited for either ambipolar bulk transport or interesting donor-acceptor photoinduced phenomena.

\section{ACKNOWLEDGMENTS}

O.G.Z. and J.B. were supported by the European Research Council (ERC) under the European Union Horizon 2020 research and innovation program (Grant Agreement No. 682539/ SOFTCHARGE). Via our membership of the UK's HEC Materials Chemistry Consortium, which is funded by EPSRC (Grant Nos. $\mathrm{EP} / \mathrm{L} 000202$ and EP/R029431), this work used the ARCHER UK National Supercomputing Service (http://www.archer.ac.uk), as well as the UK Materials and Molecular Modeling (MMM) Hub, which is partially funded by EPSRC (Grant No. EP/P020194), for computational resources. The authors also acknowledge the use of UCL Grace and Kathleen High Performance Computing Facilities.

\section{DATA AVAILABILITY}

The data that support the findings of this study are available from the corresponding author upon reasonable request.

\section{REFERENCES}

${ }^{1}$ J. W. Colson and W. R. Dichtel, Nat. Chem. 5, 453 (2013).

${ }^{2}$ N. Huang, P. Wang, and D. Jiang, Nat. Rev. Mater. 1, 16068 (2016).

${ }^{3}$ C. S. Diercks and O. M. Yaghi, Science 355, eaal1585 (2017).

${ }^{4}$ M. S. Lohse and T. Bein, Adv. Funct. Mater. 28, 1705553 (2018).

${ }^{\mathbf{5}}$ M. Dogru and T. Bein, Chem. Commun. 50, 5531 (2014).

${ }^{6}$ L. Ma, S. Wang, X. Feng, and B. Wang, Chin. Chem. Lett. 27, 1383 (2016).

${ }^{7}$ L. Yang and D.-C. Wei, Chin. Chem. Lett. 27, 1395 (2016).

${ }^{8}$ D. D. Medina, T. Sick, and T. Bein, Adv. Energy Mater. 7, 1700387 (2017).

${ }^{9}$ A. K. Mandal, J. Mahmood, and J.-B. Baek, ChemNanoMat 3, 373 (2017).

${ }^{10}$ X. Feng, L. Chen, Y. Dong, and D. Jiang, Chem. Commun. 47, 1979 (2011).

${ }^{11}$ V. S. P. K. Neti, X. Wu, S. Deng, and L. Echegoyen, CrystEngComm 15, 6892 (2013).

${ }^{12}$ S. Lin, C. S. Diercks, Y.-B. Zhang, N. Kornienko, E. M. Nichols, Y. Zhao, A. R. Paris, D. Kim, P. Yang, O. M. Yaghi, and C. J. Chang, Science 349, 1208 (2015).

${ }^{13}$ B. Sun, J. Li, W.-L. Dong, M.-L. Wu, and D. Wang, J. Phys. Chem. C 120, 14706 (2016).

${ }^{14}$ H. Wang, H. Ding, X. Meng, and C. Wang, Chin. Chem. Lett. 27, 1376 (2016).

${ }^{15}$ C. Chen, T. Joshi, H. Li, A. D. Chavez, Z. Pedramrazi, P.-N. Liu, H. Li, W. R. Dichtel, J.-L. Brédas, and M. F. Crommie, ACS Nano 12, 1936 (2018).

${ }^{16}$ X.-H. Liu, J.-Y. Yue, Y.-P. Mo, Y. Yao, C. Zeng, T. Chen, H.-J. Yan, Z.-H. Wang, D. Wang, and L.-J. Wan, J. Phys. Chem. C 120, 15753 (2016).

${ }^{17}$ B. Nath, W.-H. Li, J.-H. Huang, G.-E. Wang, Z.-h. Fu, M.-S. Yao, and G. Xu, CrystEngComm 18, 4259 (2016).

${ }^{18}$ T. Joshi, C. Chen, H. Li, C. S. Diercks, G. Wang, P. J. Waller, H. Li, J. L. Brédas, O. M. Yaghi, and M. F. Crommie, Adv. Mater. 31, 1805941 (2018).

${ }^{19}$ Z.-J. Xia, H.-C. Yang, Z. Chen, R. Z. Waldman, Y. Zhao, C. Zhang, S. N. Patel, and S. B. Darling, Adv. Mater. Interfaces 6, 1900254 (2019).

${ }^{20}$ H.-J. Zhu, M. Lu, Y.-R. Wang, S.-J. Yao, M. Zhang, Y.-H. Kan, J. Liu, Y. Chen, S.-L. Li, and Y.-Q. Lan, Nat. Commun. 11, 497 (2020).

${ }^{21}$ S. Wan, F. Gándara, A. Asano, H. Furukawa, A. Saeki, S. K. Dey, L. Liao, M. W. Ambrogio, Y. Y. Botros, X. Duan, S. Seki, J. F. Stoddart, and O. M. Yaghi, Chem. Mater. 23, 4094 (2011).

${ }^{22}$ X. Feng, L. Liu, Y. Honsho, A. Saeki, S. Seki, S. Irle, Y. Dong, A. Nagai, and D. Jiang, Angew. Chem., Int. Ed. 51, 2618 (2012).

${ }^{23}$ J. Liu, W. Zhou, J. Liu, Y. Fujimori, T. Higashino, H. Imahori, X. Jiang, J. Zhao, T. Sakurai, Y. Hattori, W. Matsuda, S. Seki, S. K. Garlapati, S. Dasgupta, E. Redel, L. Sun, and C. Wöll, J. Mater. Chem.A 4, 12739 (2016).

${ }^{24}$ N. Keller, M. Calik, D. Sharapa, H. R. Soni, P. M. Zehetmaier, S. Rager, F. Auras, A. C. Jakowetz, A. Görling, T. Clark, and T. Bein, J. Am. Chem. Soc. 140, 16544 (2018).

${ }^{25}$ T. W. Kim, S. Jun, Y. Ha, R. K. Yadav, A. Kumar, C.-Y. Yoo, I. Oh, H.-K. Lim, J. W. Shin, R. Ryoo, H. Kim, J. Kim, J.-O. Baeg, and H. Ihee, Nat. Commun. 10, 1873 (2019).

${ }^{26}$ P. Zhu and V. Meunier, J. Chem. Phys. 137, 244703 (2012).

${ }^{27}$ X. Liu, J. Tan, A. Wang, X. Zhang, and M. Zhao, Phys. Chem. Chem. Phys. 16, 23286 (2014).

${ }^{28}$ J.-J. Adjizian, P. Briddon, B. Humbert, J.-L. Duvail, P. Wagner, C. Adda, and C. Ewels, Nat. Commun. 5, 5842 (2014).

${ }^{29}$ R.-N. Wang, X.-R. Zhang, S.-F. Wang, G.-S. Fu, and J.-L. Wang, Phys. Chem. Chem. Phys. 18, 1258 (2016).

${ }^{30}$ M. Yousefi, M. Faraji, R. Asgari, and A. Z. Moshfegh, Phys. Rev. B 97, 195428 (2018).

${ }^{31}$ Y. Chen, S. Xu, Y. Xie, C. Zhong, C. Wu, and S. B. Zhang, Phys. Rev. B 98, 035135 (2018).

${ }^{32}$ S. Thomas, H. Li, and J.-L. Brédas, Adv. Mater. 31, 1900355 (2019).

${ }^{33}$ W. Jiang, H. Huang, and F. Liu, Nat. Commun. 10, 2207 (2019).

${ }^{34}$ Y. Zhou, Z. Wang, P. Yang, X. Zu, and F. Gao, J. Mater. Chem. 22, 16964 (2012).

${ }^{35}$ R. N. Gunasinghe, D. G. Reuven, K. Suggs, and X.-Q. Wang, J. Phys. Chem. Lett. 3, 3048 (2012). 
${ }^{36}$ L. Liang, P. Zhu, and V. Meunier, J. Chem. Phys. 142, 184708 (2015).

${ }^{37}$ V. Obersteiner, A. Jeindl, J. Götz, A. Perveaux, O. T. Hofmann, and E. Zojer, Adv. Mater. 29, 1700888 (2017)

${ }^{38}$ S. Thomas, H. Li, C. Zhong, M. Matsumoto, W. R. Dichtel, and J.-L. Brédas, Chem. Mater. 31, 3051 (2019).

${ }^{39}$ A. V. Kuklin, G. V. Baryshnikov, B. F. Minaev, N. Ignatova, and H. Ågren, J. Phys. Chem. C 122, 22216 (2018).

${ }^{40} \mathrm{Y}$. Jing and T. Heine, J. Am. Chem. Soc. 141, 743 (2018).

${ }^{41}$ S. Thomas, H. Li, R. R. Dasari, A. M. Evans, I. Castano, T. G. Allen, O. G. Reid, G. Rumbles, W. R. Dichtel, N. C. Gianneschi, S. R. Marder, V. Coropceanu, and J.-L. Brédas, Mater. Horiz. 6, 1868 (2019).

${ }^{42} \mathrm{~J}$. Tan, W. Li, X. He, and M. Zhao, RSC Adv. 3, 7016 (2013).

${ }^{43}$ R. Gutzler, Phys. Chem. Chem. Phys. 18, 29092 (2016).

${ }^{44}$ H. Q. Pham, D. Q. Le, N.-N. Pham-Tran, Y. Kawazoe, and D. Nguyen-Manh, RSC Adv. 9, 29440 (2019).

${ }^{45}$ B. Xu, S. Li, H. Jiao, J. Yin, Z. Liu, and W. Zhong, J. Mater. Chem.A 8, 3865 (2020).

${ }^{46}$ T. Tanaka and A. Osuka, Chem. Soc. Rev. 44, 943 (2015).

${ }^{47}$ M. D. Peeks, C. E. Tait, P. Neuhaus, G. M. Fischer, M. Hoffmann, R. Haver, A. Cnossen, J. R. Harmer, C. R. Timmel, and H. L. Anderson, J. Am. Chem. Soc. 139, 10461 (2017).

${ }^{48}$ S. Richert, B. Limburg, H. L. Anderson, and C. R. Timmel, J. Am. Chem. Soc. 139, 12003 (2017)

${ }^{49}$ E. Leary, B. Limburg, A. Alanazy, S. Sangtarash, I. Grace, K. Swada, L. J. Esdaile, M. Noori, M. T. González, G. Rubio-Bollinger, H. Sadeghi, A. Hodgson, N. Agraï, S. J. Higgins, C. J. Lambert, H. L. Anderson, and R. J. Nichols, J. Am. Chem. Soc. 140, 12877 (2018).

${ }^{50}$ Y. Kurumisawa, T. Higashino, S. Nimura, Y. Tsuji, H. Iiyama, and H. Imahori, J. Am. Chem. Soc. 141, 9910 (2019).

${ }^{51}$ V. V. Roznyatovskiy, C.-H. Lee, and J. L. Sessler, Chem. Soc. Rev. 42, 1921 (2013).

${ }^{52}$ S. Hiroto, Y. Miyake, and H. Shinokubo, Chem. Rev. 117, 2910 (2016).

${ }^{53}$ A. M. Rappe, K. M. Rabe, E. Kaxiras, and J. D. Joannopoulos, Phys. Rev. B 41, $1227(1990)$

${ }^{54}$ J. P. Perdew, K. Burke, and M. Ernzerhof, Phys. Rev. Lett. 77, 3865 (1996).

${ }^{55}$ J. P. Perdew, M. Ernzerhof, and K. Burke, J. Chem. Phys. 105, 9982 (1996)

${ }^{56}$ P. E. Blöchl, O. Jepsen, and O. K. Andersen, Phys. Rev. B 49, 16223 (1994).
${ }^{57}$ P. Giannozzi, S. Baroni, N. Bonini, M. Calandra, R. Car, C. Cavazzoni, D. Ceresoli, G. L. Chiarotti, M. Cococcioni, I. Dabo, A. Dal Corso, S. de Gironcoli, S. Fabris, G. Fratesi, R. Gebauer, U. Gerstmann, C. Gougoussis, A. Kokalj, M. Lazzeri, L. Martin-Samos, N. Marzari, F. Mauri, R. Mazzarello, S. Paolini, A. Pasquarello, L. Paulatto, C. Sbraccia, S. Scandolo, G. Sclauzero, A. P. Seitsonen, A. Smogunov, P. Umari, and R. M. Wentzcovitch, J. Phys.: Condens. Matter 21, 395502 (2009).

${ }^{58}$ A. V. Krukau, O. A. Vydrov, A. F. Izmaylov, and G. E. Scuseria, J. Chem. Phys. 125, 224106 (2006).

${ }^{59}$ S. J. Clark, M. D. Segall, C. J. Pickard, P. J. Hasnip, M. I. J. Probert, K. Refson, and M. C. Payne, Z. Kristallogr. 220, 567 (2005).

${ }^{60}$ A. D. Becke, J. Chem. Phys. 98, 5648 (1993).

${ }^{61}$ C. Lee, W. Yang, and R. G. Parr, Phys. Rev. B 37, 785 (1988).

${ }^{62}$ M. Valiev, E. J. Bylaska, N. Govind, K. Kowalski, T. P. Straatsma, H. J. J. Van Dam, D. Wang, J. Nieplocha, E. Apra, T. L. Windus, and W. A. de Jong, Comput. Phys. Commun. 181, 1477 (2010).

${ }^{63}$ J. Xi, M. Long, L. Tang, D. Wang, and Z. Shuai, Nanoscale 4, 4348 (2012).

${ }^{64}$ W. Zhang, Z. Huang, W. Zhang, and Y. Li, Nano Res. 7, 1731 (2014).

${ }^{65}$ C. Muschielok and H. Oberhofer, J. Chem. Phys. 151, 015102 (2019).

${ }^{66}$ Y. Zhao and D. G. Truhlar, J. Chem. Phys. 130, 074103 (2009).

${ }^{67}$ S. Müller and K. Müllen, Philos. Trans. R. Soc. A 365, 1453 (2007).

${ }^{68}$ Y. Saegusa, T. Ishizuka, K. Komamura, S. Shimizu, H. Kotani, N. Kobayashi, and T. Kojima, Phys. Chem. Chem. Phys. 17, 15001 (2015).

${ }^{69} \mathrm{~L}$. Piela, Ideas of Quantum Chemistry (Elsevier, 2014).

${ }^{70}$ D. Wang, L. Tang, M. Long, and Z. Shuai, J. Chem. Phys. 131, 224704 (2009).

${ }^{71}$ M. Long, L. Tang, D. Wang, Y. Li, and Z. Shuai, ACS Nano 5, 2593 (2011).

${ }^{72}$ M.-Q. Long, L. Tang, D. Wang, L. Wang, and Z. Shuai, J. Am. Chem. Soc. 131, 17728 (2009).

${ }^{73}$ Y. Cai, G. Zhang, and Y.-W. Zhang, J. Am. Chem. Soc. 136, 6269 (2014).

${ }^{74}$ J. Spencer, F. Gajdos, and J. Blumberger, J. Chem. Phys. 145, 064102 (2016).

${ }^{75}$ A. Carof, S. Giannini, and J. Blumberger, J. Chem. Phys. 147, 214113 (2017).

${ }^{76}$ S. Giannini, A. Carof, M. Ellis, H. Yang, O. G. Ziogos, S. Ghosh, and J. Blumberger, Nat. Commun. 10, 3843 (2019).

${ }^{77}$ A. Carof, S. Giannini, and J. Blumberger, Phys. Chem. Chem. Phys. 21, 26368 (2019).

${ }^{78}$ O. G. Ziogos, S. Giannini, M. Ellis, and J. Blumberger, J. Mater. Chem. C 8, 1054 (2020). 